\title{
Photoactive Catalytically Self-Threaded 2D Polyrotaxane Network for Visible Light Activated Antimicrobial Phototherapy
}

\author{
Aisan Khaligh, Rehan Khan, Duygu Deniz Akolpoğlu Başaran, Melis Özkan, and Dönüs Tuncel* \\ Cite This: ACS Appl. Polym. Mater. 2020, 2, 5726-5734 \\ Read Online
}

ABSTRACT: Here, we adapt the catalytically self-threading polyrotaxane synthesis for the construction of two-dimensional polymeric thin films using a water-oil interfacial polymerization method. In this method, the polymerization and the rotaxane formation take place simultaneously at the interface because of the presence of catalytically active cucurbit[6]uril (CB6) that can facilitate 1,3-dipolar cycloaddition reaction between alkyne and azide to form polytriazoles. By varying the concentration of the monomers, reaction time, and the size of the reaction vessel, it is possible to control the thickness and the lateral dimensions of the film. The as-synthesized film is free-floating, transparent, and robust enough to be transferred to any substrates. It contains photoactive porphyrin units which are quite appealing as a photosensitizer

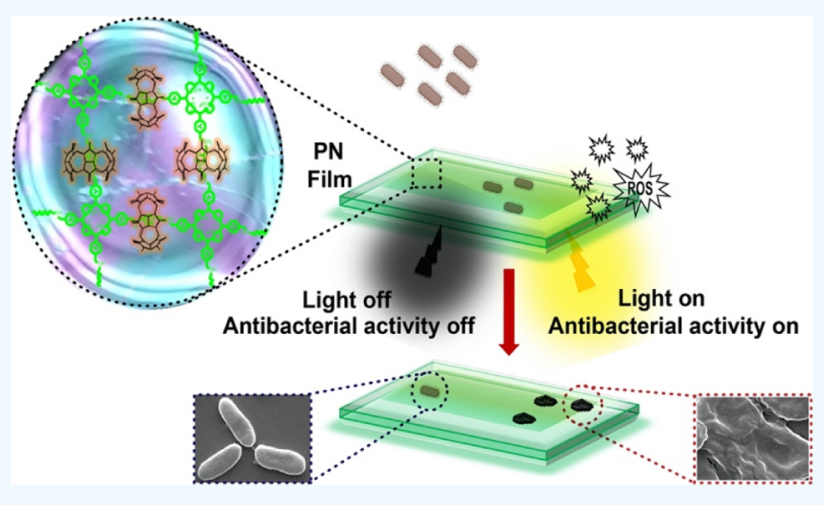
because of their capability to produce reactive oxygen species in high yield upon visible light irradiation. By taking advantage of these aforementioned features, this film was employed as a broad-spectrum photo-antimicrobial agent whose activity was switched on by light excitation against both Gram-negative and Gram-positive bacterial strains and switched off in the dark.

KEYWORDS: 2D polyrotaxane network, interfacial polymerization, free-standing transparent film, photodynamic therapy, singlet oxygen, antibacterial surface

\section{INTRODUCTION}

Two-dimensional (2D) photoactive polymeric films are highly attractive because they can be utilized in a number of different areas such as optoelectronics, separation processes, sensing, energy conversion, storage, and nanomedicine and can easily be deposited on various substrates for device fabrication owing to their film-like properties. ${ }^{1}$ In the literature, there are many efforts to synthesize $2 \mathrm{D}$ polymeric films using a number of different approaches; among them, air-liquid and liquidliquid interfacial polymerization are getting considerable attention because using these methods, large area, continuous, free-standing crystalline mono- or multilayer polymeric $2 \mathrm{D}$ films can be prepared. ${ }^{2-10}$

Porphyrin is one of the common components used in the construction of $2 \mathrm{D}$ polymeric materials because of its electroand photoactive properties and its ability to accommodate multifunctionalities required for network formation. ${ }^{11,12}$ Although the liquid-liquid interfacial polymerization was applied to the synthesis of porphyrin-based polymeric networks around $1980 \mathrm{~s}^{13-15}$ this approach remained rather dormant until recently. ${ }^{2-12}$

The examples in the literature usually involve the use of imine bond formation between the reactive multifunctional aldehydes and amine-based rigid monomers, and the resulting
2D materials are neutral and contain limited functionalities. $^{16-18}$ Ionic $2 \mathrm{D}$ polymeric films ${ }^{19}$ and the use of flexible linkers in their construction are also rather scarce. Recently, supramolecular organic frameworks and rotaxanated covalent organic frameworks have started to appear in the literature. $^{20-24}$

To expand the scope of the $2 \mathrm{D}$ polymeric free-standing films, adding further functionalities and stimuli-responsive features will be highly useful. In this regard, here, we report a free-floating 2D polyrotaxane network (PN) which possesses photoactive, rigid porphyrin cores decorated with flexible alkyl units that will act as a visible light-triggered photosensitizer as well as containing $\mathrm{pH}$-switchable rotaxanes, polytriazole, and polyamines. The thin film formed at the liquid-water interface through catalytically self-threading polyrotaxane synthesis, in which CB6 accelerates the 1,3-dipolar cycloaddition reaction between tetra-alkyne- and tetra-azide-functionalized porphyrin

Received: September 11, 2020

Accepted: November 19, 2020

Published: November 30, 2020 
monomers to form polytriazoles and, then, remains as a part of the polyrotaxane network. Although the CB6-catalyzed azidealkyne cycloaddition (CB6-AAC) reaction has been employed extensively in the synthesis of molecular switches, rotaxanes, and polyrotaxanes and the construction of nanostructured materials, ${ }^{25-30}$ here, for the first time, this approach has been explored in the synthesis of free-standing, photoactive 2D polyrotaxanated thin films. PN also has a high potential to be employed as a light-triggered antibacterial wound dressing as well as photodynamic coatings for the delicate medical equipment as bacterial infections and the rapid evolution of antibiotic-resistant pathogens continue to pose a severe threat to human health, and the alternative therapeutical strategies are urgently needed to combat the antimicrobial resistance problem. ${ }^{31,32}$ In this context, utilizing photodynamic therapy (PDT) is highly appealing because PDT is a clinically approved, effective, and noninvasive treatment mode which is based on the excitation of a photosensitizer with a specific wavelength of light to generate highly toxic reactive oxygen species (ROS) including singlet oxygen from the molecular oxygen to induce microbial killing. ${ }^{33,34}$ In this regard, our porphyrin-cored PN film satisfies the requirements of an ideal photosensitizer with its ability to generate ROS in high yield. It shows high inhibitory activity against both Gram-positive and Gram-negative bacteria via visible-light-triggered production of ROS.

\section{EXPERIMENTAL SECTION}

2.1. Materials. Propargyl amine, 2-chloroethylammonium chloride, sodium azide, pyrrole, chloroform, and dichloromethane as well as all the other chemicals and reagents used for organic syntheses were of analytical grade and obtained from Sigma-Aldrich, unless otherwise stated. 2,7-Dichlorofluorescein diacetate (DCFH-DA) used in ROS measurement was purchased from Sigma-Aldrich. The deuterated solvents used in NMR were purchased from Merck. Thin layer chromatography (TLC) was performed on silica gel plates (Kieselgel $60 \mathrm{~F} 254,1 \mathrm{~mm})$. Ultrapure deionized water $(R \geq 18 \mathrm{M} \Omega$ $\mathrm{cm}^{-1}$ ) from the Milli-Q plus water purification system (Millipore, Bedford, MA, USA) was used throughout the study. Escherichia coli DH5- $\alpha$ strain (Gram-negative) and Bacillus subtilis MTCC 441 strain (Gram-positive) were used for bacteria assays. Yeast extract, sodium chloride, tryptone, and agar powder used for the preparation of liquid lysogeny broth (LB) as nutrient medium and semi-solid LB agar were obtained from Sigma-Aldrich. Glutaraldehyde solution used in scanning electron microscopy (SEM) imaging of bacteria was obtained from Merck. 10X phosphate-buffered saline (PBS) used in bacteria assays was purchased from Sigma-Aldrich. To make $1 \times$ PBS, the $10 \times$ stock solution was diluted 10 -fold with deionized water and then autoclaved.

2.2. Instrumentation. ${ }^{1} \mathrm{H}$ NMR and ${ }^{13} \mathrm{C}$ NMR spectra were acquired using a Bruker AVANCE III $400 \mathrm{MHz}$ NMR spectrometer. Mass spectra were recorded on an Agilent 6210 LC/MS TOF mass spectrometer. The morphology studies were performed using transmission electron microscopy (TEM, FEI Tecnai G2 F30) and SEM (Quanta 200 FEG). Cross-sectional images of the PN film layer were taken using focused ion beam/SEM (FIB/SEM, FEI NanoSEM dual beam system). The thickness of the PN films was also attained via atomic force microscopy (AFM, AFM Park, XE100). Raman spectra were recorded using a confocal Raman microscope (WITec Alpha 300R). The Thermo Scientific FLASH 2000 CHNS analyzer was used for elemental analysis of the sample. Fourier transform infrared (FT-IR) spectra were obtained using a Bruker Alpha-II Platinum ATR FT-IR spectrometer. All optical characterizations were performed by the Cary $300 \mathrm{UV}$-vis spectrophotometer. X-ray diffraction (XRD) patterns were recorded using an X-ray diffractometer [X'pert pro MPD (PANalytical)]. A TA Instruments Q500 thermogravimetric analyzer was used to measure the thermal stability of the compound. Chemical and elemental analysis were determined using X-ray photoelectron spectroscopy (XPS) (Thermo Fisher Scientific). Measurements were performed with a spot size of $\sim 400$ $\mu \mathrm{m}$, a pass energy of $30 \mathrm{eV}$, and a step size of $0.1 \mathrm{eV}$. Fluorescence spectra were recorded using the Cary Eclipse fluorescence spectrophotometer (Agilent Technologies, USA).

2.3. Synthesis and Characterization of Porphyrin Precursors. 5,10,15,20-Tetrakis $(\alpha$-bromo- $p$-tolyl)porphyrin (TPP-4Br), 2 azidoethylamine, and $N, N^{\prime}, N^{\prime \prime}, N^{\prime \prime \prime}-((((9 E, 10 Z, 14 Z, 16 E, 19 Z)-5 H, 6 H-$ porphyrin-5,10,15,20-tetrayl)tetrakis(benzene-4,1 diyl)) tetrakis (methylene))tetrakis(prop-2-yn-1-amine) (TPP-4AL) were synthesized according to the literature procedures. ${ }^{28,29}$

2.3.1. Synthesis of $N_{,} N^{\prime}, N^{\prime \prime}, N^{\prime \prime \prime}-(((19 E, 10 Z, 14 Z, 16 E, 19 Z)-5 H, 6 H-$ Porphyrin-5, 10, 15,20-tetrayl)tetrakis(benzene-4,1-diyl))tetrakis(methylene))tetrakis(2-azidoethan-1-amine), (TPP-4AZ). TPP-4Br $(0.21 \mathrm{~g}, 0.21 \mathrm{mmol})$ was dissolved in chloroform $(5 \mathrm{~mL})$, and the resulting solution was added dropwise to the stirring solution of 2azidoethylamine $(1.5 \mathrm{~g}, 17.42 \mathrm{mmol})$ in chloroform $(3 \mathrm{~mL})$. The reaction mixture was stirred at RT for $24 \mathrm{~h}$. Completion of the reaction was confirmed by TLC. After the reaction was over, the solvent was removed under reduced pressure to obtain a solid residue which was washed with water several times. A purple-colored precipitate was collected and dried in vacuo. Yield: $0.205 \mathrm{~g}, 95 \%$.

${ }^{1} \mathrm{H}$ NMR (400 MHz, $\left.\mathrm{CDCl}_{3}\right): \delta-2.74(\mathrm{~s}, 2 \mathrm{H}), 1.60(\mathrm{~s}), 3.11(\mathrm{t}$, $\left.8 \mathrm{H},{ }^{3} J_{\mathrm{HH}}=5.6 \mathrm{~Hz}\right), 3.62\left(\mathrm{t}, 8 \mathrm{H},{ }^{3} J_{\mathrm{HH}}=5.6 \mathrm{~Hz}\right), 4.15(\mathrm{~s}, 8 \mathrm{H}), 7.74(\mathrm{~d}$, $\left.8 \mathrm{H},{ }^{3} \mathrm{~J}_{\mathrm{HH}}=8.0 \mathrm{~Hz}\right), 8.20\left(\mathrm{~d}, 8 \mathrm{H},{ }^{3} \mathrm{~J}_{\mathrm{HH}}=8.0 \mathrm{~Hz}\right), 8.88(\mathrm{~s}, 8 \mathrm{H}) ;{ }^{13} \mathrm{C}$ NMR $\left(100 \mathrm{MHz}, \mathrm{D}_{2} \mathrm{O}\right): \delta 48.4,51.6,53.7,119.9,126.4,131.0,134.7$, 139.4, 141.0; ES-MS $(m / z)$ : calcd for $\mathrm{C}_{56} \mathrm{H}_{55} \mathrm{~N}_{20}[\mathrm{M}+\mathrm{H}]^{+}$, 1007.47; found, 1007.49 .

2.3.2. Synthesis of Hydrochloride Salt of TPP-4AZ. TPP-4AZ $(0.250 \mathrm{mg}, 0.248 \mathrm{mmol})$ was first dissolved in $1 \mathrm{M} \mathrm{HCl}$ (aq) solution and stirred at room temperature for $15 \mathrm{~min}$. The solvent was then removed under reduced pressure to obtain a solid residue which was washed with acetone. The precipitate was redissolved in water, and the solution was subjected to freeze-drying to obtain fluffy powder. Yield: $0.268 \mathrm{mg}, 95 \%$.

UV-vis $\left(\mathrm{H}_{2} \mathrm{O}, \mathrm{nm}\right), \lambda_{\max }: 415,520,554,593,648 ;{ }^{1} \mathrm{H}$ NMR $(400$ $\left.\mathrm{MHz}, \mathrm{D}_{2} \mathrm{O}\right): \delta 3.59$ (b, $\left.8 \mathrm{H}\right), 3.99(\mathrm{~b}, 8 \mathrm{H}), 4.79(\mathrm{~s}, 8 \mathrm{H}), 8.23(\mathrm{~b}, 8$ $\mathrm{H}), 8.88(\mathrm{~b}, 8 \mathrm{H}), 9.04(\mathrm{~s}, 8 \mathrm{H})$.

2.4. General Procedure for the Synthesis of the 2D Polyrotaxane Network (PN). PN as a free-standing, 2D polyrotaxanated thin film was synthesized by interfacial polymerization at the interface between oil and aqueous phases. For a typical synthesis, Th oil phase was prepared by dissolving TPP-4AL $(2.2 \mathrm{mg}$, $\left.2.5 \times 10^{-3} \mathrm{mmol}\right)$ in $10 \mathrm{~mL}$ of chloroform. To prepare the aqueous phase, TPP-4AZ $\left(2.5 \mathrm{mg}, 2.5 \times 10^{-3} \mathrm{mmol}\right)$ and CB6 $(10 \mathrm{mg}, 10 \times$ $10^{-3} \mathrm{mmol}$ ) were separately dissolved in $1 \mathrm{M} \mathrm{HCl}$ (aq) (each in 5 $\mathrm{mL}$ ). CB6 solution was added to the solution of TPP-4AZ. The mixture was stirred for about $5 \mathrm{~min}$ to obtain a homogeneous solution. The oil phase was placed into a $50 \mathrm{~mL}$ beaker, and the aqueous phase was added carefully on top of the oil phase. The beaker was closed with a Petri dish and set aside to allow for film formation through interfacial polymerization.

Elemental analysis is calculated for one repeating unit of $\mathbf{P N}$, $\mathrm{C}_{375} \mathrm{H}_{589} \mathrm{~N}_{140} \mathrm{O}_{148}{ }^{+20}$ (ex. $100 \mathrm{H}_{2} \mathrm{O}$ ), (\%): C, 47.78; H, 6.30; N, 20.80 . Found: C, 47.77; H, 4.94; N, 21.11.

2.5. ROS Measurements. DCFH-DA assay was carried out as reported in the literature. ${ }^{30,35}$ Specifically, $0.5 \mathrm{~mL}$ of DCFH-DA solution in ethanol $(1 \mathrm{mM})$ was mixed with $2 \mathrm{~mL}$ of aqueous $\mathrm{NaOH}$ solution $(0.01 \mathrm{M})$ for $30 \mathrm{~min}$ at ambient conditions for transformation into nonfluorescent $2^{\prime}, 7^{\prime}$-dichlorodihydrofluorescein (DCFH). The resulting hydrolysate was then mixed with $10 \mathrm{~mL}$ of sodium phosphate buffer $(25 \mathrm{mM}, \mathrm{pH} 7.4)$ and kept cold $\left(2{ }^{\circ} \mathrm{C}\right)$ in a dark place until use. The final concentration of DCFH solution was $40 \mu \mathrm{M}$. Blank solution was prepared by diluting $0.5 \mathrm{~mL}$ of $40 \mu \mathrm{M}$ DCFH with $1 \mathrm{~mL}$ of water. The solution was irradiated under white light $\left(1 \mathrm{~mW} \mathrm{~cm}{ }^{-2}\right)$ for $4 \mathrm{~min}$. The emission spectra of DCF solution were recorded in a 498-800 $\mathrm{nm}$ emission range every $1 \mathrm{~min}$ with an excitation wavelength of 488 . The same procedure as blank measurements was followed for PN. 
Scheme 1. Molecular Structures of Alkyne- and Azide-Substituted Porphyrin Monomers and the Proposed Structure of a Repeating Unit of the 2D Polyrotaxane Network
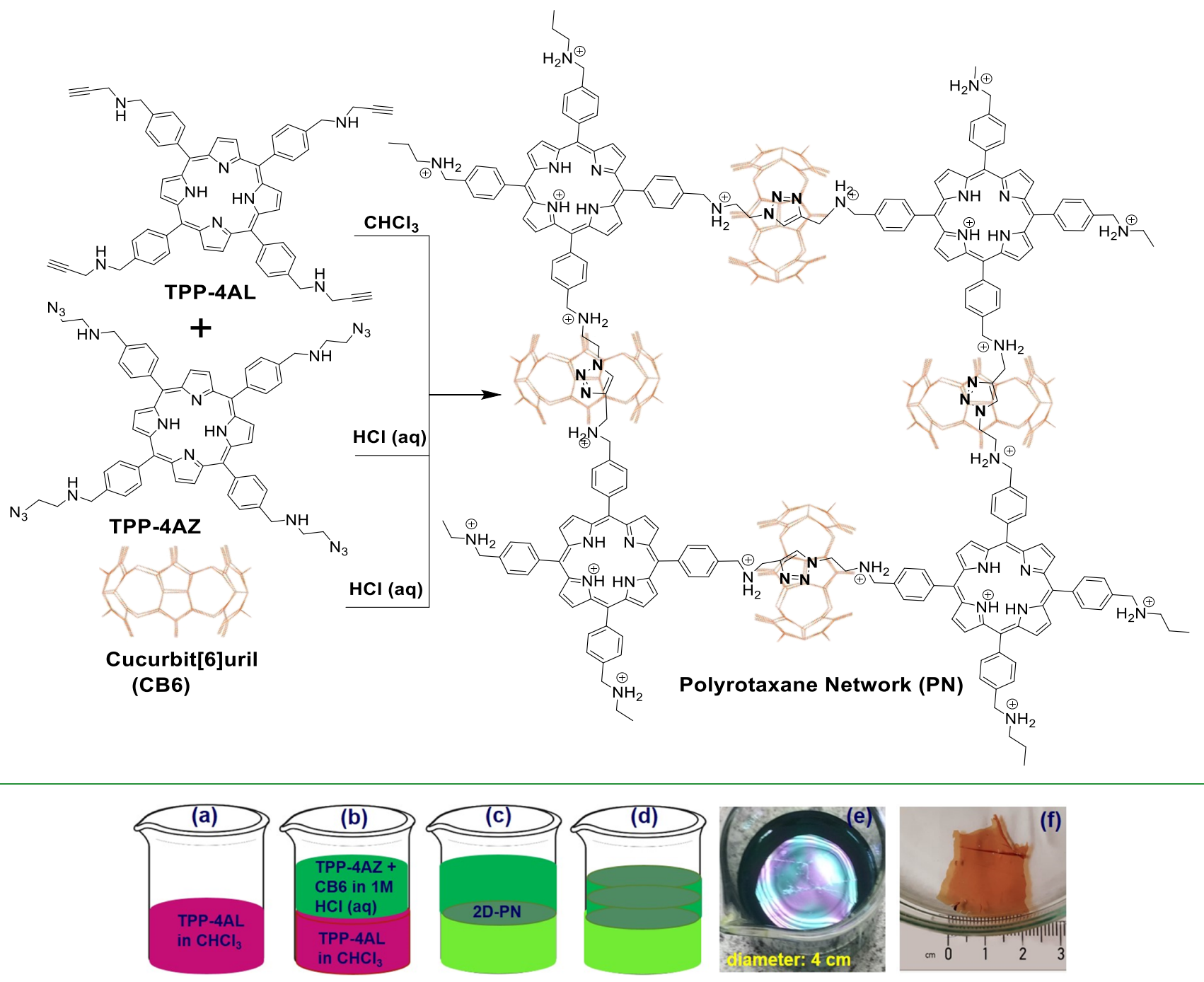

Figure 1. Cartoon representation of the interfacial synthesis of the 2D polyrotaxane network: (a) solution of TPP-4AL in chloroform; (b) aqueous solution of TPP-4AZ and CB6 in $1 \mathrm{M} \mathrm{HCl}$ layered onto TPP-4AL solution; (c) formation of a 2D polyrotaxane network (PN) at the interface; (d) PN monolayer which is less dense than the water, floating to the top layer to form stacked layers; (e) the photograph of interference of the top layer on the beaker; (f) free-floating PN film in water.

2.6. Testing Visible Light-Triggered Antibacterial Activity. A single colony of E. coli (DH5- $\alpha$ ) and B. subtilis (MTCC-441) on a solid LB agar plate was selected and transferred to liquid LB culture medium and grown overnight $\left(37^{\circ} \mathrm{C}, 200 \mathrm{rpm}\right)$. Bacteria were harvested with centrifugation $\left(4^{\circ} \mathrm{C}, 5000 \mathrm{rpm}, 5 \mathrm{~min}\right)$ and washed by PBS solution (10 mM, pH 7.4). $20 \mu \mathrm{L}$ of suspension of bacteria was added on $0.7 \mathrm{~cm} \times 0.7 \mathrm{~cm}$ PN film-coated glass and then irradiated upon white light with a flux of $22 \mathrm{~mW} \mathrm{~m}^{-2}$ for $30 \mathrm{~min}$. Bacteria-added PN films were transferred to the $1 \mathrm{~mL}$ LB culture medium and incubated for $2 \mathrm{~h}$ at $37{ }^{\circ} \mathrm{C}$ with $200 \mathrm{rpm}$. A $50 \mu \mathrm{L}$ of resulting bacterial mixture was spread on the solid LB agar plate. The colonies formed after overnight incubation at $37^{\circ} \mathrm{C}$ and quantified. This procedure was repeated in the dark without exposure to light, and control experiments were carried out with glass. Survival of bacteria on PN after exposed to white light illumination $\left(22 \mathrm{~mW} \mathrm{~cm}{ }^{-2}, 30\right.$ min) was determined by a surface plating method, and colony forming units (cfu) of E. coli and B. subtilis were evaluated by using the following equation

$$
\frac{\mathrm{cfu}}{\mathrm{mL}}=\frac{\text { no. } \text { of colonies } \times \text { dilution factor }}{\text { volume of culture plate }}
$$

2.7. Preparation of SEM Samples for the Investigation of Morphological Changes upon PN Treatment. Prior to the treatment, silica wafers $(1 \mathrm{~cm} \times 1 \mathrm{~cm})$ were incubated for 30 min with 2-propanol and then with ethanol for the removal of organic contamination. Wafers were completely dried in a $37{ }^{\circ} \mathrm{C}$ incubator. For the treatment groups, wafers were coated with $\mathbf{P N}$ and sterilized under an UV lamp for $5 \mathrm{~min}$. All the wafers were transferred to a 6well plate. Suspensions of E. coli cultures in $\mathrm{LB}\left(30 \mathrm{~mL}, \mathrm{OD}_{600} \sim 0.5\right)$ were spread evenly onto silica wafers (for control groups) and PNcoated silica wafers. The light group was exposed to white light (22 $\mathrm{mW} \mathrm{cm}^{-2}, 30 \mathrm{~min}$ ), whereas the dark group was kept in the dark room for $30 \mathrm{~min}$. Cells were fixed onto silica wafers using $2.5 \%$ glutaraldehyde solution in PBS for overnight at $4{ }^{\circ} \mathrm{C}$. After that, wafers containing cells were washed with PBS, pure water, $25 \%(\mathrm{v} / \mathrm{v})$ ethanol, $50 \%$ ethanol, $75 \%$ ethanol, and finally pure ethanol for $2 \mathrm{~min}$ with each solvent. The wafers were dried using a critical point dryer. Dry samples were coated with $5 \mathrm{~nm}$ of $\mathrm{Au} / \mathrm{Pd}$ alloy, and images were captured at $15 \mathrm{kV}$. 


\section{RESULTS AND DISCUSSION}

3.1. Synthesis and Characterization of the 2D Polyrotaxane Network (PN Film). Scheme 1 shows the molecular structures of monomers and a repeating unit of the 2D polyrotaxane network (PN). CB6, TPP-4Br, and TPP4AL were synthesized according to the previously reported procedures. $^{28,29}$ TPP-4AZ was synthesized by treating TPP$4 \mathrm{Br}$ with excess 2 -azido-ethylamine at room temperature in very good yield (around 95\%). TPP-4AZ was characterized using spectroscopic techniques including ${ }^{1} \mathrm{H}$ and ${ }^{13} \mathrm{C} N M R$, FT-IR, UV-vis, and ESI-mass (Figures S1-S8).

With azide- and alkyne-functionalized porphyrin monomers in hand, we next set out to synthesize the $2 \mathrm{D}$ polyrotaxane network (PN) through the well-established CB6-catalyzed azide alkyne cycloaddition (CB-AAC) reaction as illustrated in Figure 1. To prepare the aqueous phase, TPP-4AZ (1 equimolar) and CB6 (4 equimolar) were dissolved in $1 \mathrm{M}$ aqueous $\mathrm{HCl}$ solution. By dissolving TPP-4AL (1 equimolar) in chloroform, the oil phase was obtained. The concentrations of the monomers were kept between 0.05 and $0.5 \mathrm{mM}$ to study the concentration effect on the film thickness. The aqueous phase was layered onto the oil phase carefully in a beaker or a Petri dish with varying surface areas. PN formed at the interface through water-oil interfacial polymerization but floated on the top of the beaker after around $6 \mathrm{~h}$ (with monomers concentration of $0.5 \mathrm{mM}$ ) that can also be inferred from the colorful thin film interference (Figure 1e). The same result was achieved only after $24 \mathrm{~h}$ with low monomer concentrations $(0.05 \mathrm{mM})$. The top layer PN film could be easily harvested by a tweezer or transferred onto a substrate by immersing them horizontally into the aqueous phase of the reaction mixture. Herein, the PN film is adhered well over the surface of various substrates such as glass, silicon wafer, filter paper, plastic, and so forth after drying for $10 \mathrm{~min}$ at room temperature (see Movie S1, Supporting Information). After each harvesting process, the fresh PN film kept forming on the surface of the aqueous phase of the reaction mixture. When the PN film is transferred into a Petri dish containing water, it floats (see Movie S2, Supporting Information). The fresh harvested film is initially green colored but after washing with water, the color becomes rose-pink (Figure 1f), indicating the deprotonation of pyrrolic nitrogens of porphyrin cores. Keeping all the reaction conditions the same but in the absence of CB6, no film formation and no light interference on the surface were observed. Moreover, the presence of other $\mathbf{C B}$ homologues (CB7 and CB8) also failed to form the film. These results clearly show (wide infra) the formation of the polyrotaxane network through the well-known catalytic ability of CB6 for 1,3-dipolar cycloaddition reaction between azideand alkyne-substituted monomers as reported previously. ${ }^{25-30}$ Although the film is insoluble in any solvent, we managed to disperse it in DMSO after prolonged stirring at room temperature and recorded its ${ }^{1} \mathrm{H}$ NMR spectrum to further prove the formation of the polyrotaxane network. The ${ }^{1} \mathrm{H}$ NMR spectrum displays clearly the characteristic signals for the protons of CB6, porphyrin, and phenyl and, most importantly, the signals for the triazole protons around 6.5$7.0 \mathrm{ppm}$, indicating formation of rotaxane and the encapsulation of triazole in the cavity of CB6 (Figure S9). Elemental analysis also provides some evidence by agreeing with the calculated elemental compositions of $\mathrm{C}, \mathrm{H}$, and $\mathrm{N}$ for a proposed repeating unit of the polyrotaxane network.
Figure 2 compares FT-IR spectra of PN with its monomers. The appearance of the carbonyl stretching signal at $1735 \mathrm{~cm}^{-1}$

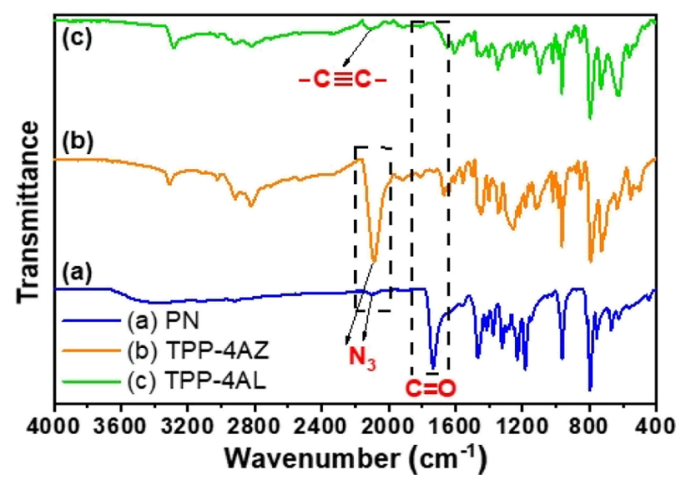

Figure 2. Comparison of the FT-IR spectra of PN and its monomers: (a) PN, (b) TPP-4AZ, and (c) TPP-4AL.

along with the decrease in the intensity of azide and alkyne signals at 2095 and $2130 \mathrm{~cm}^{-1}$, respectively, in the FT-IR spectrum of the developed PN assembly compared to those TPP-4AZ and TPP-4AL monomers further confirmed the success of the CB6-AAC reaction. The FT-IR spectrum of CB6 is also presented in Figure S10.

In order to gain insights into how the reaction time affects the thickness of the PN film, a series of samples were prepared (with a monomer concentration of $0.5 \mathrm{mM}$ ) and films were transferred over silicon substrates by regular time intervals to record their SEM images (Figure S11). Results showed that the PN thickness was gradually increased over time as 75,422 , 1056, and $1693 \mathrm{~nm}$ after 3, 12, 24, and $72 \mathrm{~h}$, respectively. Using the same procedure, SEM images of the films harvested after 24 and $48 \mathrm{~h}$ were recorded as around 18 and $30 \mathrm{~nm}$, respectively, with low monomer concentrations of $0.05 \mathrm{mM}$ (Figure S12). The thicknesses of PN films prepared with monomer concentrations of $0.5 \mathrm{mM}$ and harvested after 7 and $13 \mathrm{~h}$ determined by AFM are about 120 and $450 \mathrm{~nm}$, respectively (Figure S13). These results are in good agreement with SEM results and prove that by varying the concentration of monomers and the reaction time, it would be possible to control the thickness of the film. Figure 3 shows the TEM images of $\mathbf{P N}$ after $3 \mathrm{~h}$ reaction. From the images, it can be seen that the film is multilayered and thin and has a smooth surface.

We were unable to obtain the XRD spectrum of the PN film; therefore, powder was prepared by drying of the harvested film for overnight at room temperature, and its XRD analysis was then carried out. According to the XRD spectrum, PN is crystalline with well-defined diffraction peaks at $2 \theta=7.8,15.3$,
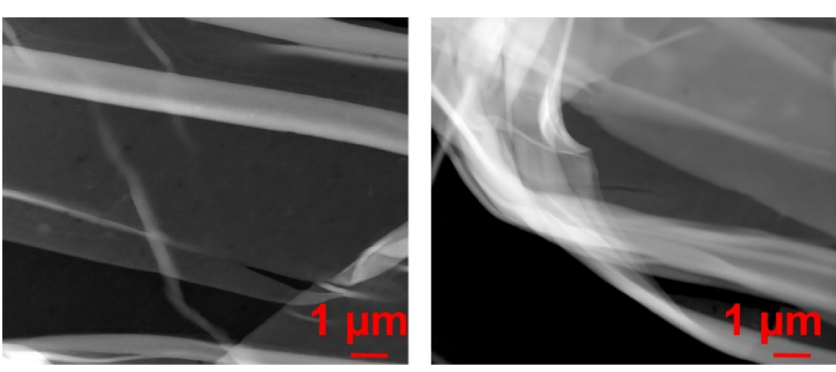

Figure 3. TEM images of PN. 
and $22.9^{\circ}$ (Figure S14). The Raman spectrum for PN powder (Figure S15) was also recorded. The spectrum reveals the characteristic $\mathrm{D}$ and $\mathrm{G}$ bands at 1343 and $1575 \mathrm{~cm}^{-1}$, respectively, with the intensity ratio of $I_{\mathrm{D}} / I_{\mathrm{G}}$ as 0.94 . These results look similar to the Raman spectrum of the polyrotaxanated $\mathrm{COF}$ film $\left(\mathrm{T}_{\mathrm{P}} \mathrm{VCB}[7]\right)$ reported by Das et al. $^{23}$

Chemical analysis of the PN film was also carried out by XPS analysis (Figure S16). The high-resolution N 1s spectrum presents a peak at around $400 \mathrm{eV}$, which was deconvoluted into several peaks with binding energies of 400.3, 401.3, and $399.1 \mathrm{eV}$ and can be assigned as $\mathrm{N}-\mathrm{N}=\mathrm{C}$ (triazole- $\mathrm{N}$ ) and $\mathrm{C}-\mathrm{NH}$ (amine- $\mathrm{N}$ ) and $\mathrm{N}(\mathrm{C})_{3}$ and $\mathrm{C}=\mathrm{N}-\mathrm{C}$ (pyrrolic-N), respectively. The $\mathrm{C} 1 \mathrm{~s}$ XPS spectrum was fitted into six components 284, 284.7, 285.4, 286.2, 287.9, and $289.4 \mathrm{eV}$ corresponding to the $\mathrm{C}=\mathrm{C}, \mathrm{C}-\mathrm{C}, \mathrm{C}-\mathrm{N}, \mathrm{C}=\mathrm{N}, \mathrm{C}=\mathrm{O}$, and $\mathrm{N}-\mathrm{C}=\mathrm{O}$ bonds, respectively. The $\mathrm{O} 1 \mathrm{~s}$ XPS spectrum of $\mathbf{P N}$ exhibited one major peak around $532 \mathrm{eV}$ assigned to the $\mathrm{C}=\mathrm{O}$ bond.

Thermal stability of $\mathbf{P N}$ powder was investigated with thermogravimetric analysis (TGA) in the range of $25-950{ }^{\circ} \mathrm{C}$ with a rate of $10{ }^{\circ} \mathrm{C} \mathrm{min}{ }^{-1}$ under a nitrogen atmosphere. Its thermogram shows three decomposition steps (Figure S17). An initial mass loss (below $200{ }^{\circ} \mathrm{C}, 9.1 \%$ ) is attributed to the elimination of physically adsorbed water molecules. The subsequent step started around $280{ }^{\circ} \mathrm{C}$ probably due to the degradation of triazole, aliphatic linkages, and CB6s. Even at around $450{ }^{\circ} \mathrm{C}$, only $50 \%$ of weight loss was observed indicating the high stability of PN.

The PN film harvested after $15 \mathrm{~h}$ with around $650 \mathrm{~nm}$ thickness (measured by FIB/SEM, Figure S18) was transferred onto a quartz slide $(1 \times 1 \mathrm{~cm})$, and its absorption and transmission spectra were recorded as in its green-colored protonated form and pink-rose colored free base which was obtained after washing with an aqueous alkaline solution. If we compare their UV-vis absorption spectra (Figure 4a), the

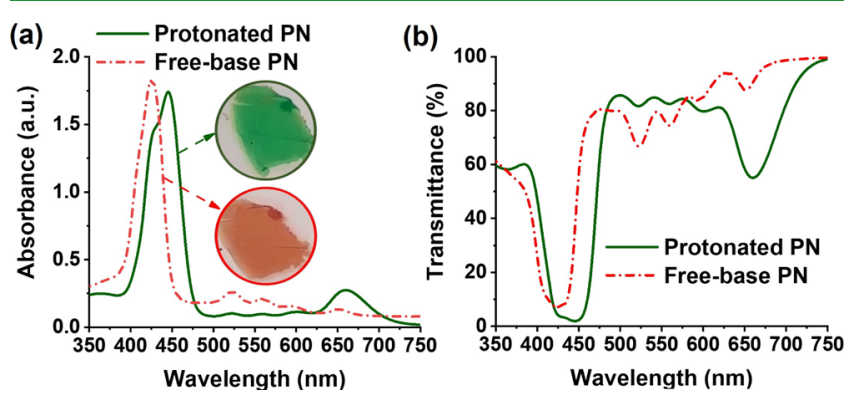

Figure 4. UV-vis spectra of the PN film layer before and after treatment with $0.1 \mathrm{M}$ aqueous $\mathrm{KOH}$ solution: (a) absorbance, (b) transmittance.

Soret band around $450 \mathrm{~nm}$ in the spectrum of protonated PN shifts about $20 \mathrm{~nm}$ to the blue region, a band around $670 \mathrm{~nm}$ decreases significantly, and four weak $Q$ bands appear for the free-base PN. The PN film in both states also shows transparency and transmit the light (around 80\%) in the visible region $(450-650 \mathrm{~nm}$ ), as shown in Figure $4 \mathrm{~b}$. These features make the $\mathbf{P N}$ film as a very promising photoactive layer for many applications. The optical band gap energies $\left(E_{\mathrm{g}}\right)$ of free-base PN and its monomers, TPP-4AZ and TPP-4AL, are estimated through their UV-vis Tauc plots as 2.78, 2.76, and $2.79 \mathrm{eV}$, respectively (Figure S19).
3.2. Investigation of the ROS Generation Ability of PN. After authentication of the structure of the targeted PN film by detailed characterizations, we set out to evaluate its visible-light-induced ROS generation capacity because its photodynamic antimicrobial activity depends mainly on how efficiently the ROS are produced. The ROS generation ability of PN was probed using a well-established DCFH-DA assay under low fluence of white light $\left(1 \mathrm{~mW} \mathrm{~cm}^{-2}\right)$ and ambient conditions. $^{30,35}$ In this study, the thin PN film coated on the quartz slide $(1 \mathrm{~cm} \times 1 \mathrm{~cm})$ was transferred to the lower half of a quartz cuvette filled with diluted DCFH solution which was obtained by the hydrolysis of DCFH-DA in the alkaline media. It should be noted that DCFH is nonfluorescent, but it becomes fluorescent by oxidation in the presence of ROS (Figure 5a). We also studied the ROS generation ability of

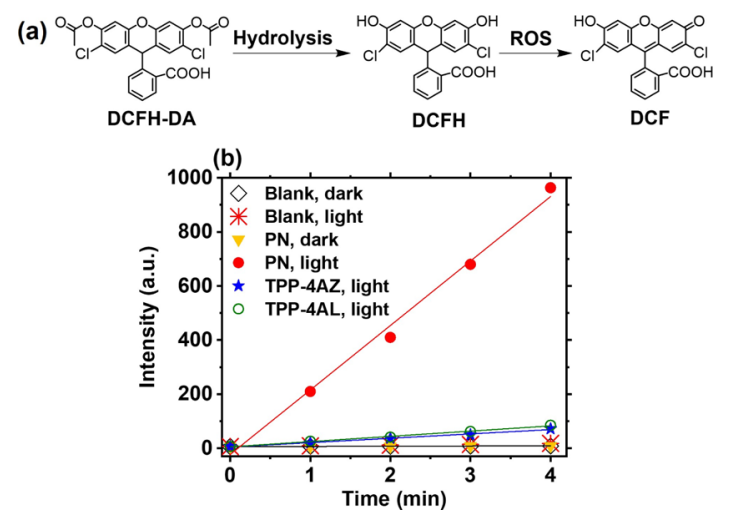

Figure 5. (a) Mechanism of ROS detection using DCFH-DA. (b) Time response curves of DCFH oxidation with and without PN under white light, with and without PN in dark, with TPP monomers under white light.

TPP-4AZ and TPP-4AL monomers using the same procedure by coating $40 \mu \mathrm{L}$ of each monomer solution $\left(2.5 \times 10^{-3} \mathrm{mmol}\right.$, $10 \mathrm{~mL}$ chloroform) over the quartz slide and drying at RT. Figure $5 b$ compares the time response curves of DCFH oxidation in the presence and absence of PN and TPP monomers under white light and in the dark. The corresponding fluorescence spectra can also be seen in Figure $\mathrm{S} 20 \mathrm{a}-\mathrm{f}$. The fluorescence intensity of DCF at $524 \mathrm{~nm}$ was significantly enhanced in the presence of PN over 4 min whitelight illumination, whereas very weak emission bands were observed in the case of $\mathbf{P N}$ in dark and control experiments (in the absence of PN under light and in dark). TPP-4AL and TPP-4AZ monomers showed very low ROS production compared to PN. These results confirm that the PN film, as an efficient photosensitizer, has a remarkable light-triggered ROS generation ability under white-light illumination even with relatively low light intensity.

3.3. Evaluation of Visible Light-Triggered Antimicrobial Activity of PN. Encouraged by the ability of PN to generate ROS efficiently, we next wished to explore its visible light-induced antibacterial activity on the representative Gramnegative and Gram-positive bacteria, namely, E. coli and B. subtilis, respectively. The antimicrobial effect of $\mathbf{P N}$ was investigated by analyzing cfu of both Gram-negative and Grampositive bacteria after overnight incubation with and without light treatment. For the phototoxicity experiments, treatment of the bacteria through a white light with a flux of $22 \mathrm{~mW} \mathrm{~cm}^{-2}$ for $30 \mathrm{~min}$ was found to be optimal. Initially, the growth of 


\section{B. subtilis}
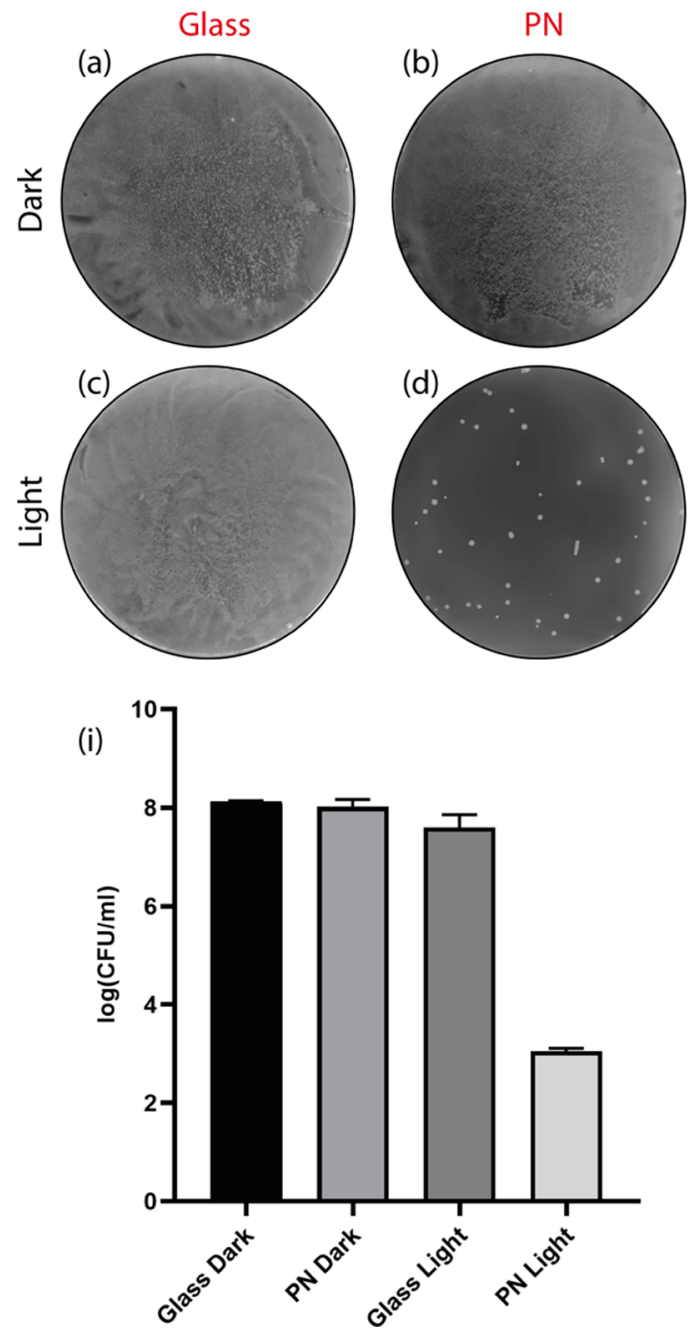

E. coli
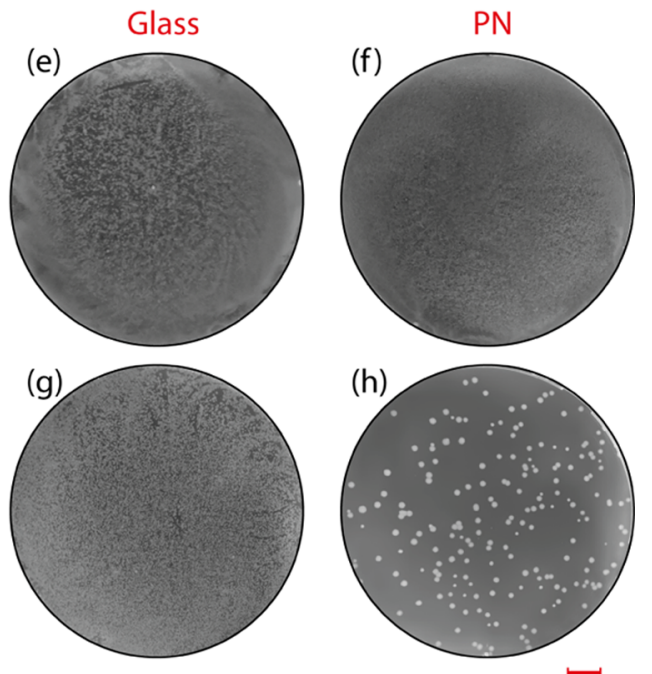

$10 \mathrm{~mm}$

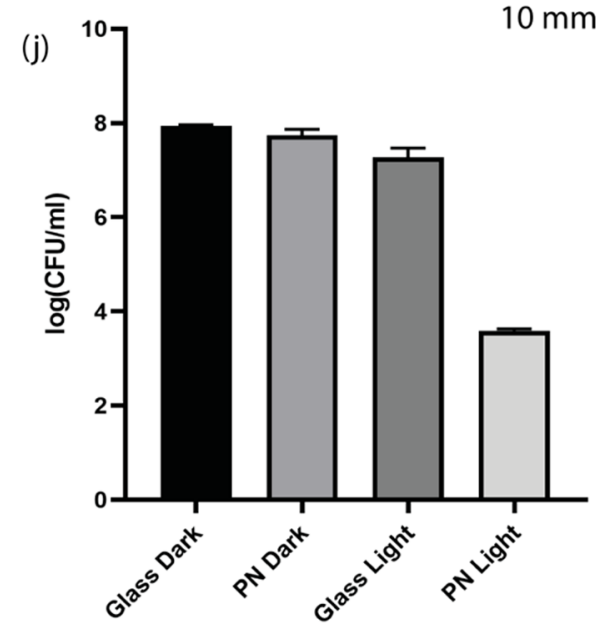

Figure 6. Plate photographs of bacteria on the agar plate: B. subtilis (a) on glass, (b) on PN in the dark, (c) on glass, (d) on PN under light illumination; and E. coli (e) on glass, (f) on PN in the dark, (g) on glass, (h) on PN under light illumination. Antibacterial activity of the PN film toward (i) B. subtilis and (j) E. coli in the dark and under light illumination. Light treatment: white light with a flux of $22 \mathrm{~mW} \mathrm{~cm}^{-2}$ for $30 \mathrm{~min}$. The data represented the mean \pm standard deviation of three separate experiments.

bacteria on the PN in the dark and under light as well as the control sets (in the absence of PN) was examined at a high concentration of bacteria. The results showed that the agar plates of the control groups of bacteria in the dark and under light as well as the sample coated with PN and not treated with light were covered densely with bacteria (Figure $6 \mathrm{a}-\mathrm{c}, \mathrm{e}-\mathrm{g}$ ). On the contrary, there were noticeably fewer colonies on the light exposed PN under the same conditions (Figure 6d,h). In order to obtain the data of the control groups in Figure 6i,j, bacteria were diluted $10^{4}$ times and new sets of samples were prepared. In the dilution case, the colonies on the plate were clearly countable (Figure S21). Bacteria on light-exposed PN showed more than 4-log reduction compared to other groups for both E. coli and B. subtilis (Figure 6i,j). This means that bacteria on the light-exposed PN film showed at least $10^{4}$ times less growth than bacteria on PN in the dark and control sets. It is promising to observe a high growth inhibition rate using white light only within $30 \mathrm{~min}$ because the antibacterial effect of previously constructed porphyrin-based MOF films was obtained after $3 \mathrm{~h}$ exposure to blue light. ${ }^{36}$ It is also observed that PN shows slightly higher phototoxicity toward B. subtilis than E. coli. The difference between two types of bacteria can be attributed to the less negatively charged surface of Gramnegative, E. coli, compared to Gram-positive, B. subtilis. ${ }^{37}$

Furthermore, in order to show the $\mathrm{pH}$ effect on $E$. coli and $B$. subtilis, $\mathrm{PBS}$ having $\mathrm{pH} 5$ and $\mathrm{pH} 9$ was used. It is observed that in the acidic and basic media, even in the absence of PN, the survival rate of both bacteria types decreased in the dark and light, but under light and in the presence of PN, no colony formation was observed in both acidic and basic media compared to the one in the neutral medium. The quantitative analysis diagrams (Figure S22) of bacteria survival rates and the corresponding agar plate photographs (Figures S23 and S24) are presented in the Supporting Information.

3.4. Visualizing PN-Bacteria Interaction under SEM. A strong inhibitory effect of $\mathbf{P N}$ on the bacteria was further verified by visualizing its interaction with $E$. coli using SEM. SEM is a powerful imaging technique to investigate the morphological changes of the treated and nontreated control groups of bacteria. Control samples were handled on silica wafers, whereas PN-coated silica wafers were used for treatment groups in the dark and under white light irradiation. 

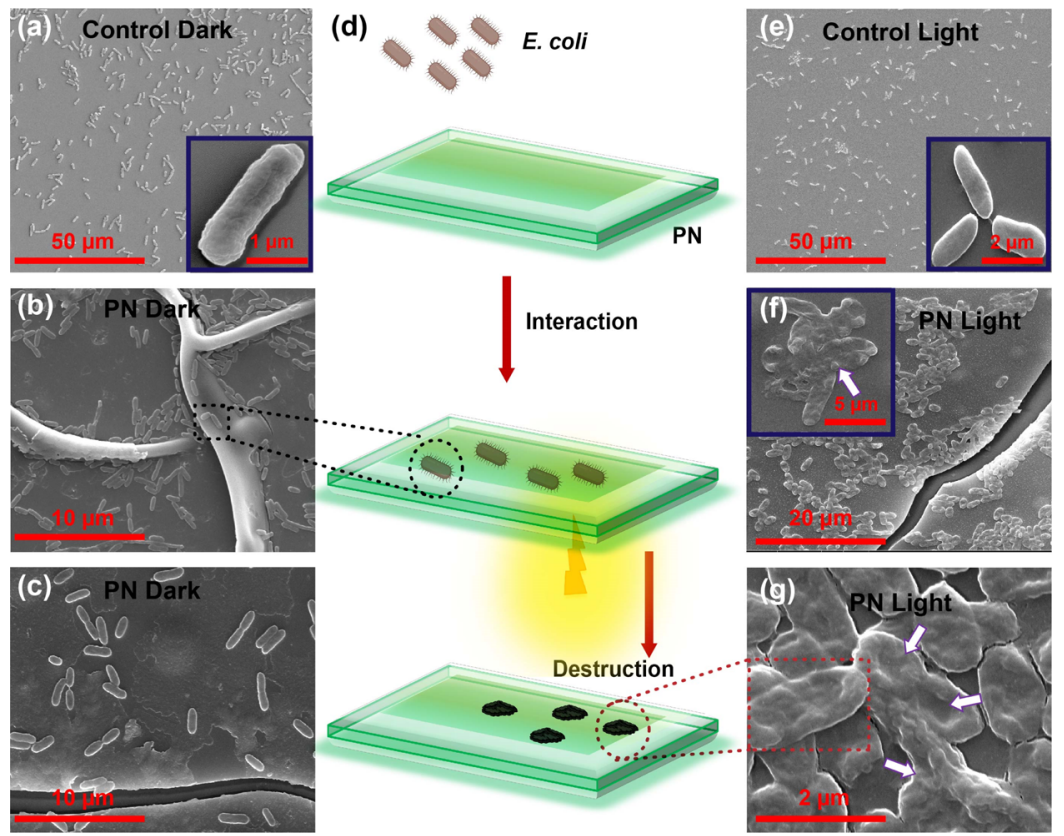

Figure 7. SEM images of E. coli (a) on glass, (b,c) on PN in the dark. (d) Schematic representation of PN and E. coli interaction. (e) SEM images of $E$. coli on glass, $(\mathrm{f}, \mathrm{g})$ on $\mathbf{P N}$ under light illumination.

As can be clearly seen from Figure 7a,e, control samples possess a uniform and rod-shaped phenotype with a smooth surface both in the dark and under white light illumination. PN-treated cultures in the dark (Figure $7 \mathrm{~b}, \mathrm{c}$ ) appeared to be having similar morphological properties with control groups maintaining the structure of cellular membranes without any damage and floating on the surface of PN. On the other hand, upon light exposure, bacteria on $\mathbf{P N}$ were found to be shrunken (Figure $7 f, g$ ). Their membranes were ruptured, and leakage of the cytoplasm and cellular organelles was observed and pointed by white arrows. In Figure $7 \mathrm{~d}$, the schematic representation of action of $\mathbf{P N}$ is illustrated.

\section{CONCLUSIONS}

In conclusion, we reported a new 2D free floating, photoactive polyrotaxane-based network which was synthesized through CB6-AAC of two properly functionalized porphyrin derivatives by a facile interfacial polymerization method at room temperature. While the thickness of the film can be controlled by varying the concentration of the monomers and the reaction time, the area of the PN film can only be limited by the size of the reaction flask used. In the absence of CB6 (even in the presence of $\mathrm{CB} 7$ and $\mathrm{CB} 8$ ), the film fails to form. The resulting thin film floats in water and is transparent. It can also be transferred onto any desired substrates and adhered well onto the surfaces after drying. It also appears to be a good photosensitizer with an ability to generate ROS even under low fluence of visible light and, consequently, proved itself as an efficient broad-spectrum antibacterial agent against both Gram-positive and Gram-negative bacteria. Another important feature of $\mathbf{P N}$ is that it has controllable disguise because its antimicrobial activity was only switched on upon light irradiation and switched off in the dark. PN is very promising for the development of stable photodynamic antimicrobial coatings including wound dressing and healing applications. Currently, we are working toward this direction.

\section{ASSOCIATED CONTENT}

Supporting Information

The Supporting Information is available free of charge at https://pubs.acs.org/doi/10.1021/acsapm.0c01010.

Adherence PN film over the surface of various substrates (MP4)

Floating PN film after being transferred into a Petri dish containing water (MP4)

${ }^{1} \mathrm{H}$ NMR spectrum of 2 -azido-ethylamine; ${ }^{1} \mathrm{H}$ NMR, ${ }^{13} \mathrm{C}$ NMR, ESI-MS and FT-IR spectra of TPP-4AZ; ${ }^{1} \mathrm{H}$ NMR, UV-vis absorbance and fluorescence spectra of hydrochloride salt of TPP-4AZ and TPP-4AL; ${ }^{1} \mathrm{H}$ NMR spectrum of PN; FT-IR spectra of CB6; thickness study of PN film versus reaction time using SEM cross-section view; SEM images of PN film harvested at 48 and $72 \mathrm{~h}$ (monomer concentrations $0.05 \mathrm{mM}$ ); AFM images of PN film harvested at 7 and $13 \mathrm{~h}$; XRD, Raman and XPS spectra of PN, TGA graph of PN; FIB/SEM crosssection image of PN; Tauc plots of $\mathbf{P N}$ and its porphyrin monomers; PL spectra of DCF at $524 \mathrm{~nm}$ with and without PN under white-light illumination and dark as well as with porphyrin monomers under light; and plate photographs of $B$. subtilis and E. coli with $10^{4}$ dilution on agar plate in the light and under dark (PDF)

\section{AUTHOR INFORMATION}

\section{Corresponding Author}

Dönüs Tuncel - Institute of Materials Science and Nanotechnology, National Nanotechnology Research Center (UNAM) and Department of Chemistry, Bilkent University, 06800 Ankara, Turkey; 이이이.org/0000-0001-7762-

9200; Email: dtuncel@fen.bilkent.edu.tr

\section{Authors}

Aisan Khaligh - Institute of Materials Science and Nanotechnology, National Nanotechnology Research Center 
(UNAM) and Department of Chemistry, Bilkent University, 06800 Ankara, Turkey

Rehan Khan - Institute of Materials Science and Nanotechnology, National Nanotechnology Research Center (UNAM) and Department of Chemistry, Bilkent University, 06800 Ankara, Turkey

Duygu Deniz Akolpoğlu Başaran - Institute of Materials Science and Nanotechnology, National Nanotechnology Research Center (UNAM), Bilkent University, 06800 Ankara, Turkey

Melis Özkan - Institute of Materials Science and Nanotechnology, National Nanotechnology Research Center (UNAM), Bilkent University, 06800 Ankara, Turkey

Complete contact information is available at:

https://pubs.acs.org/10.1021/acsapm.0c01010

\section{Notes}

The authors declare no competing financial interest.

\section{ACKNOWLEDGMENTS}

We thank S. E. Hadi for his assistance to take some of the SEM images of PN film.

\section{ABBREVIATIONS}

NMR, nuclear magnetic resonance; FT-IR, Fourier-transform infrared spectroscopy; TGA, thermogravimetric analysis; SEM, scanning electron microscopy; TEM, transmission electron microscopy; FIB, focused ion beam; AFM, atomic force microscopy; XRD, X-ray diffraction; XPS, X-ray photoelectron spectroscopy; UV-vis, ultraviolet-visible; ESI-MS, electrospray ionization mass spectrometry; ROS, reactive oxygen species; PN, 2D polyrotaxane network; TPP-4AL, tetra-propyl amine-functionalized porphyrin; TPP-4AZ, tetra-azido aminefunctionalized porphyrin; TPP-4Br, 5,10,15,20-tetrakis $(\alpha$ bromo-p-tolyl)porphyrin; CB6, cucurbit[6]uril; DCFH-DA, 2,7-dichlorofluorescein diacetate; LB, lysogeny broth; E. coli, Escherichia coli; B. subtilis, Bacillus subtilis

\section{REFERENCES}

(1) Dong, R.; Zhang, T.; Feng, X. Interface-Assisted Synthesis of 2D Materials: Trend and Challenges. Chem. Rev. 2018, 118, 6189-6235.

(2) Matsumoto, M.; Valentino, L.; Stiehl, G. M.; Balch, H. B.; Corcos, A. R.; Wang, F.; Ralph, D. C.; Mariñas, B. J.; Dichtel, W. R. Lewis-Acid-Catalyzed Interfacial Polymerization of Covalent Organic Framework Films. Chem 2018, 4, 308-317.

(3) Müller, V.; Hinaut, A.; Moradi, M.; Baljozovic, M.; Jung, T. A.; Shahgaldian, P.; Möhwald, H.; Hofer, G.; Kröger, M.; King, B. T.; Meyer, E.; Glatzel, T.; Schlüter, A. D. A Two-Dimensional Polymer Synthesized at the Air/Water Interface. Angew. Chem., Int. Ed. 2018, 57, 10584-10588.

(4) Payamyar, P.; Kaja, K.; Ruiz-Vargas, C.; Stemmer, A.; Murray, D. J.; Johnson, C. J.; King, B. T.; Schiffmann, F.; VandeVondele, J.; Renn, A.; Götzinger, S.; Ceroni, P.; Schütz, A.; Lee, L.-T.; Zheng, Z.; Sakamoto, J.; Schlüter, A. D. Synthesis of a Covalent Monolayer Sheet by Photochemical Anthracene Dimerization at the Air/Water Interface and Its Mechanical Characterization by AFM Indentation. Adv. Mater. 2014, 26, 2052-2058.

(5) Murray, D. J.; Patterson, D. D.; Payamyar, P.; Bhola, R.; Song, W.; Lackinger, M.; Schlüter, A. D.; King, B. T. Large Area Synthesis of a Nanoporous Two-Dimensional Polymer at the Air/Water Interface. J. Am. Chem. Soc. 2015, 137, 3450-3453.

(6) Müller, V.; Shao, F.; Baljozovic, M.; Moradi, M.; Zhang, Y.; Jung, T.; Thompson, W. B.; King, B. T.; Zenobi, R.; Schlüter, A. D. Structural Characterization of a Covalent Monolayer Sheet Obtained by Two-Dimensional Polymerization at an Air/Water Interface. Angew. Chem., Int. Ed. 2017, 56, 15262-15266.

(7) Dong, R.; Han, P.; Arora, H.; Ballabio, M.; Karakus, M.; Zhang, Z.; Shekhar, C.; Adler, P.; Petkov, P. S.; Erbe, A.; Mannsfeld, S. C. B.; Felser, C.; Heine, T.; Bonn, M.; Feng, X.; Cánovas, E. High-mobility Band-like Charge Transport in a Semiconducting Two-Dimensional Metal-Organic Framework. Nat. Mater. 2018, 17, 1027.

(8) Bauer, T.; Zheng, Z.; Renn, A.; Enning, R.; Stemmer, A.; Sakamoto, J.; Schlüter, A. D. Synthesis of Free-Standing, Monolayered Organometallic Sheets at the Air/Water Interface. Angew. Chem., Int. Ed. 2011, 50, 7879-7884.

(9) Dey, K.; Pal, M.; Rout, K. C.; Kunjattu, H. S.; Das, A.; Mukherjee, R.; Kharul, U. K.; Banerjee, R. Selective Molecular Separation by Interfacially Crystallized Covalent Organic Framework Thin Films. J. Am. Chem. Soc. 2017, 139, 13083-13091.

(10) Zhang, N.; Wang, T.; Wu, X.; Jiang, C.; Zhang, T.; Jin, B.; Ji, H.; Bai, W.; Bai, R. From 1D Polymers to 2D Polymers: Preparation of Free-Standing Single-Monomer-Thick Two-Dimensional Conjugated Polymers in Water. ACS Nano 2017, 11, 7223-7229.

(11) Liu, K.; Qi, H.; Dong, R.; Shivhare, R.; Addicoat, M.; Zhang, T.; Sahabudeen, H.; Heine, T.; Mannsfeld, S.; Kaiser, U.; Zheng, Z.; Feng, X. On-Water Surface Synthesis of Crystalline, Few-Layer TwoDimensional Polymers Assisted by Surfactant Monolayers. Nat. Chem. 2019, 11, 994-1000.

(12) Zhong, Y.; Cheng, B.; Park, C.; Ray, A.; Brown, S.; Mujid, F.; Lee, J.-U.; Zhou, H.; Suh, J.; Lee, K.-H.; Mannix, A. J.; Kang, K.; Sibener, S. J.; Muller, D. A.; Park, J. Wafer-Scale Synthesis of Monolayer Two-Dimensional Porphyrin Polymers for Hybrid Superlattices. Science 2019, 366, 1379-1384.

(13) Wen, L.; Li, M.; Schlenoff, J. B. Polyporphyrin Thin Films From the Interfacial Polymerization of Mercaptoporphyrins. J. Am. Chem. Soc. 1997, 119, 7726-7733.

(14) Wamser, C. C.; Bard, R. R.; Senthilathipan, V.; Anderson, V. C.; Yates, J. A.; Lonsdale, H. K.; Rayfield, G. W.; Friesen, D. T.; Lorenz, D. A. Synthesis and Photoactivity of Chemically Asymmetric Polymeric Porphyrin Films Made by Interfacial Polymerization. J. Am. Chem. Soc. 1989, 111, 8485-8491.

(15) Li, W.; Wamser, C. C. Synthesis and Characterization of Interfacially Polymerized Films of Tetraphenylporphyrin Derivatives. Langmuir 1995, 11, 4061-4071.

(16) Sahabudeen, H.; Qi, H.; Glatz, B. A.; Tranca, D.; Dong, R.; Hou, Y.; Zhang, T.; Kuttner, C.; Lehnert, T.; Seifert, G. Wafer-Sized Multifunctional Polyimine-Based Two-Dimensional Conjugated Polymers with High Mechanical Stiffness. Nat. Commun. 2016, 7, 13461.

(17) Sheberla, D.; Sun, L.; Blood-Forsythe, M. A.; Er, S.; Wade, C. R.; Brozek, C. K.; Aspuru-Guzik, A.; Dincă, M. High Electrical Conductivity in $\mathrm{Ni}_{3}(2,3,6,7,10,11 \text {-hexaiminotriphenylene) })_{2}$, a Semiconducting Metal-Organic Graphene Analogue. J. Am. Chem. Soc. 2014, 136, 8859-8862.

(18) Dong, R.; Pfeffermann, M.; Liang, H.; Zheng, Z.; Zhu, X.; Zhang, J.; Feng, X. Large-area, Free-standing, Two-dimensional Supramolecular Polymer Single-Layer Sheets for Highly Efficient Electrocatalytic Hydrogen Evolution. Angew. Chem., Int. Ed. 2015, 54, $12058-12063$.

(19) Huang, N.; Wang, P.; Addicoat, M. A.; Heine, T.; Jiang, D. Ionic Covalent Organic Frameworks: Design of a Charged Interface Aligned on 1D Channel Walls and Unusual Electrostatic Functions. Angew. Chem., Int. Ed. 2017, 56, 4982-4986.

(20) Zhang, K.-D.; Tian, J.; Hanifi, D.; Zhang, Y.; Sue, A. C.-H.; Zhou, T.-Y.; Zhang, L.; Zhao, X.; Liu, Y.; Li, Z.-T. Toward a SingleLayer Two-Dimensional Honeycomb Supramolecular Organic Framework in Water. J. Am. Chem. Soc. 2013, 135, 17913-17918.

(21) Tian, J.; Chen, L.; Zhang, D.-W.; Liu, Y.; Li, Z.-T. Supramolecular Organic Frameworks: Engineering Periodicity in Water Through Host-Guest Chemistry. Chem. Commun. 2016, 52, 6351-6362.

(22) Zhang, X.; Nie, C.-B.; Zhou, T.-Y.; Qi, Q.-Y.; Fu, J.; Wang, X.Z.; Dai, L.; Chen, Y.; Zhao, X. The Construction of Single-layer Two- 
Dimensional Supramolecular Organic Frameworks in Water through the Self-Assembly of Rigid Vertexes and Flexible Edges. Polym. Chem. 2015, 6, 1923-1927.

(23) Das, G.; Sharma, S. K.; Prakasam, T.; Gándara, F.; Mathew, R.; Alkhatib, N.; Saleh, N. i.; Pasricha, R.; Olsen, J.-C.; Baias, M. A Polyrotaxanated Covalent Organic Network Based on Viologen and Cucurbit[7]uril. Commun. Chem. 2019, 2, 106.

(24) Pfeffermann, M.; Dong, R.; Graf, R.; Zajaczkowski, W.; Gorelik, T.; Pisula, W.; Narita, A.; Müllen, K.; Feng, X. Free-Standing Monolayer Two-Dimensional Supramolecular Organic Framework with Good Internal Order. J. Am. Chem. Soc. 2015, 137, 1452514532.

(25) Tuncel, D.; Steinke, J. H. G. Catalytic Self-threading: a New Route for the Synthesis of Polyrotaxanes. Macromolecules 2004, 37, 288-302.

(26) Tuncel, D. n. s.; Tiftik, H. B.; Salih, B. pH-Responsive Polypseudorotaxane Synthesized through Cucurbit[6] uril Catalyzed 1, 3-dipolar Cycloaddition. J. Mater. Chem. 2006, 16, 3291-3296.

(27) Tuncel, D.; Özsar, Ö.; Tiftik, H. B.; Salih, B. Molecular Switch Based on a Cucurbit[6]uril Containing Bistable [3]Rotaxane. Chem. Commun. 2007, 1369-1371.

(28) Tuncel, D.; Ünal, Ö.; Artar, M. Supramolecular Assemblies Constructed by Cucurbituril-Catalyzed Click Reaction. Isr. J. Chem. 2011, 51, 525-532.

(29) Tuncel, D.; Cindir, N.; Koldemir, Ü. [5]Rotaxane and [5]Pseudorotaxane Based on Cucurbit[6] uril and Anchored to a Meso-tetraphenyl Porphyrin. J. Inclusion Phenom. Macrocyclic Chem. 2006, 55, 373-380.

(30) Özkan, M.; Keser, Y.; Hadi, S. E.; Tuncel, D. A [5]RotaxaneBased Photosensitizer for Photodynamic Therapy. Eur. J. Org. Chem. 2019, 3534-3541.

(31) Gupta, A.; Mumtaz, S.; Li, C.-H.; Hussain, I.; Rotello, V. M. Combatting Antibiotic-Resistant Bacteria using Nanomaterials. Chem. Soc. Rev. 2019, 48, 415-427.

(32) Li, X.; Bai, H.; Yang, Y.; Yoon, J.; Wang, S.; Zhang, X. Supramolecular Antibacterial Materials for Combatting Antibiotic Resistance. Adv. Mater. 2019, 31, 1805092.

(33) Wilson, B. C.; Patterson, M. S. The Physics, Biophysics and Technology of Photodynamic Therapy. Phys. Med. Biol. 2008, 53, R61.

(34) Phillips, D. Light Relief: Photochemistry and Medicine. Photochem. Photobiol. Sci. 2010, 9, 1589-1596.

(35) Bourré, L.; Thibaut, S.; Briffaud, A.; Rousset, N.; Eléouet, S.; Lajat, Y.; Patrice, T. Indirect Detection of Photosensitizer ex vivo. J. Photochem. Photobiol., B 2002, 67, 23-31.

(36) Zhou, W.; Begum, S.; Wang, Z.; Krolla, P.; Wagner, D.; Bräse, S.; Wöll, C.; Tsotsalas, M. High Antimicrobial Activity of MetalOrganic Framework-Templated Porphyrin Polymer Thin Films. ACS Appl. Mater. Interfaces 2018, 10, 1528-1533.

(37) Ren, X.; Yang, C.; Zhang, L.; Li, S.; Shi, S.; Wang, R.; Zhang, X.; Yue, T.; Sun, J.; Wang, J. Copper Metal-Organic Frameworks Loaded on Chitosan Film for the Efficient Inhibition of Bacteria and Local Infection Therapy. Nanoscale 2019, 11, 11830-11838. 